\title{
Mendelian randomization informs shared genetic etiology underlying exposure and outcome by interrogating correlated horizontal pleiotropy
}

\section{Qing Cheng}

Duke NUS Graduate Medical School

Lin Chen ( $\square$ Ichen@health.bsd.uchicago.edu )

University of Chicago

Jin Liu

jin.liu@duke-nus.edu.sg https://orcid.org/0000-0002-5707-2078

\section{Article}

Keywords: Mendelian randomization (MR), correlated horizontal pleiotropy (CHP), genetic etiology

Posted Date: September 30th, 2021

DOI: https://doi.org/10.21203/rs.3.rs-860357/v1

License: (c) (i) This work is licensed under a Creative Commons Attribution 4.0 International License.

Read Full License

Version of Record: A version of this preprint was published at Nature Communications on October 30th, 2022. See the published version at https://doi.org/10.1038/s41467-022-34164-1. 
- Mendelian randomization informs shared genetic etiology

\begin{abstract}
Mendelian randomization (MR) harnesses genetic variants as instrumental variables (IVs) to study the causal effect of exposure on trait/disease using summary statistics from genome-wide association studies. Classic MR assumptions are violated when IVs are associated with unmeasured confounders, i.e., when correlated horizontal pleiotropy (CHP) arises. Such confounders could be a shared gene or inter-connected pathways underlying exposure and outcome. We propose MR-CUE (MR with Correlated horizontal pleiotropy Unraveling shared Etiology and confounding), for estimating causal effect while identifying IVs with CHP and accounting for estimation uncertainty. For those IVs, we map their cis-associated genes and enriched pathways to inform shared genetic etiology underlying exposure and outcome. We apply MR-CUE to study the effects of interleukin 6 on multiple traits/diseases and identify several $S 100$ genes involved in cross-traits etiology. We assess the effects of multiple exposures on type 2 diabetes across European and East Asian populations.
\end{abstract}

\title{
Introduction
}

In the post-genome-wide association study (GWAS) era, many efforts were made to step beyond genetic associations towards causation and mechanistic examinations. Mendelian randomization (MR) assesses the causal effect of potential risk exposures on outcome traits and diseases by leveraging genetic variants as instrument variables (IV) and integrating existing GWAS summary statistics $^{1}$. MR has been widely applied to study the relationships among complex traits and

${ }^{*}$ Correspondence should be addressed to Lin S. Chen (lchen@health.bsd.uchicago.edu) and Jin Liu (jin.liu@duke-nus.edu.sg) 
diseases, and have achieved numerous successes in providing causal evaluations and suggesting disease prevention and therapeutic strategies ${ }^{2}$.

Two-sample MR methods take as input two sets of summary statistics, IV-to-exposure and IV-to-outcome association statistics, to estimate the causal effect of exposure on outcome. Since genotypes are 'Mendelian randomized' during meiosis, they are generally unrelated to the external unmeasured confounding factors. Classic MR methods imposed strong assumptions on the validity of IVs. They assumed IVs to be associated with the exposure ("relevance"); to affect the outcome only through the exposure ("exclusion restriction"); and to be unconfounded ("exchangeability"). Fig. 1 1 illustrated the classic assumptions. However, those assumptions are often challenged by the pervasiveness of horizontal pleiotropy — genetic variants affecting outcome via other pathways independent of exposure. The presence of horizontal pleiotropy can bias the estimation and confound the causal inference if not properly handled. Specifically, the 'uncorrelated horizontal pleiotropy (UHP)' is a phenomenon where a genetic variant affects outcome via other pathways not through exposure, and 'correlated horizontal pleiotropy (CHP)' is a phenomenon where a genetic variant affects both exposure and outcome through a heritable shared factor, i.e., an IV being associated with unmeasured confounders. In the recent literature, many robust MR methods were proposed to relax IV assumptions and allow for IVs with UHP either by treating those IVs as outliers $\$ 3$, or by modeling their UHP effects in a joint mode ${ }^{5}\left[\frac{8}{6}\right.$. More recently, other MR methods $\$ \sqrt[9]{10}$, were developed to estimate and adjust for both uncorrelated and correlated horizontal pleiotropy. As a stepwise method, CAUSE ${ }^{9}$ first identifies the IVs with CHP effects, and subsequently estimates the causal effect of exposure on outcome by fixing the parameters related to CHP. The method cML-MA ${ }^{10}$ used a constrained maximum likelihood to draw causal inference by excluding IVs with either UHP or CHP. Both methods allowed only IVs in weak or no linkage disequilibrium (LD).

Correlated horizontal pleiotropy is a challenging phenomenon to address, yet frequently arises in MR analyses. Many complex traits/diseases are polygenic, i.e., affected by multiple genes and pathways, and some common genes/pathways may be shared between exposure and outcome. When a genetic variant being associated with the genes/pathways is selected as an IV , CHP is present and it builds a confounded relationship between exposure and outcome. Fig. 1 b-c shows an example in which correlated horizontal pleiotropy is present between body mass index (BMI) and triglycerides (TG). Without properly identifying and accounting for CHP, the effect of exposure on outcome could be confounded and the estimated causal effect of outcome on exposure is also non-zero, i.e., reverse causation may occur. To properly study causation and identify the effect of CHP, as illustrated in Fig. 1 $\mathrm{d}$ right panel we propose that the effect of confounders on outcome $\left(\alpha_{k}\right)$ can be decomposed into two parts: the first part is the shared confounding effect across all IVs with CHP and is proportional to the confounders' effect on exposure induced by each IV; and the second part $\left(\widetilde{\alpha}_{k}\right)$ captures how IV-specific perturbation to confounders (a variant's unique effect on a set of confounder genes/pathways) may affect outcome, and is orthogonal to the first part. Existing methods 10 considered only the first part and implicitly assumed that IVs with CHP affect the confounders via a deterministic common mechanism. In contrast, our method also allows for IV-specific perturbations to confounder sets, and can capture more complicated CHP effects when multiple underlying pathways and confounders exist. When estimating the effect from exposure on outcome, CHP induces a bias, $\delta=E\left(\frac{\delta \gamma_{k}+\widetilde{\alpha}_{k}}{\gamma_{k}}\right)$. The bias is equal to the shared CHP effect parameter on outcome, $\delta$. When 
IVs with CHP have bi-directional effects and the effects are balanced with a mean of zero, the bias is zero. If unbalanced CHP is present $(\delta \neq 0)$ and unadjusted, the effect of exposure on outcome is given by $\beta_{2}=\beta_{1}+\delta$, and is non-zero even if the true causal effect $\beta_{1}$ is zero. False positives may arise or power may be reduced. As a side note, IVs with CHP tend to be involved in inter-connected pathways and be associated with common drivers in the pathways. Their correlations to those common drivers may further lead to a non-zero genetic correlation that describes the relationship between two traits/diseases. Therefore, a non-zero genetic correlation $^{11}$ between two traits/diseases may reflect the extent of pleiotropic genes/pathways rather than a true causal relationship 12 .

Properly accounting for CHP induced genetic confounding is of utmost importance in making valid causal inference in MR analyses, though the task is often not easy. It is commonly assumed in the literature that not all selected IVs are affected by genetic confounding. Thus, selected IVs can be partitioned into two sets as depicted in Fig. 11. Variants in IV Set 1 (left panel, only UHP) are not associated with unmeasured confounders while variants in IV Set 2 (right panel, with CHP) are associated with the unknown confounding gene(s)/pathway(s). Existing method ${ }^{9}$ first deconvolutes the normal mixture distribution of pleiotropic effects and identifies the IV 'membership' (from Set 1 or 2) for each IV, and then estimates the causal effect. In this work, we propose a MR method, MR-CUE (MR with Correlated horizontal pleiotropy Unraveling shared Etiology and confounding). MR-CUE also allows all IVs to have potential non-zero UHP effects. It builds a Bayesian hierarchical model to estimate the causal effect while simultaneously quantifying the probability of each IV being affected by CHP. Since CHP effects could be highly or moderately sparse, the quantification of estimation uncertainty by MR-CUE allows us to identify IVs with CHP at different levels of confidence and make precise causal inference with the proper control of statistical significance.

Another major innovation is that we propose to further study sets of IVs with CHP and examine their cis-associated genes and involved pathways. Through two examples, we illustrate that the estimated IVs/variants with CHP can suggest genes and pathways that are suspected sources of genetic confounding. Those genes and pathways may shed light on the shared genetic etiology for traits and diseases affected by a common exposure, or may reveal relevant pathways and mechanisms underlying different causal exposures for a complex disease outcome. Those disease-relevant common confounders and pathways could inform concerted mechanisms and etiologies across populations and ethnic groups.

[Figure 1 about here.]

\section{Results}

\section{MR-CUE examines causal effects by delineating correlated and un- correlated horizontal pleiotropic effects}

We propose MR-CUE to estimate the causal effect from exposure $(X)$ on outcome $(Y)$ while accounting for both uncorrelated and correlated horizontal pleiotropy. As illustrated in Fig. 11d, we model the IV-to-outcome effect of the $k$-th IV $(k=1, \ldots, p), \Gamma_{k}$, as a function of IV-toexposure effect, $\gamma_{k}$, and pleiotropic effects: 


$$
\Gamma_{k}=\beta_{1} \gamma_{k}+\theta_{k}+\alpha_{k}, \quad k=1, \ldots, p .
$$

where $\beta_{1}$ is the causal effect of exposure on outcome; $\theta_{k}$ is the UHP effect, and $\alpha_{k}$ is the CHP effect of the $k$-th IV; and both the IV-to-outcome and IV-to-exposure effects, $\Gamma_{k}$ and $\gamma_{k}$, respectively, can be obtained from GWASs. We assume that all IVs may have UHP effects, $\theta_{k}$, while only a proportion of IVs may also have CHP effects. As illustrated in Fig. 1 right panel, we also assume that for an IV with CHP, it affects the exposure and confounder proportionally, and $\gamma_{k}$ is the sum of IV-to-exposure effect via and not via confounders. We rescale the IV-to-confounder effect to be 1 and the effect of confounders on exposure is then $\gamma_{k}$. In Fig. 1 $\mathrm{d}$, the line representing the direct effect from IV to exposure is omitted to avoid over-parameterization since it is assumed to change proportionally with IV-to-confounder effect. By definition, the CHP effect, $\alpha_{k}$, is correlated with IV-to-exposure effect $\gamma_{k}$. Different than existing method ${ }^{9}$ assuming CHP effect being always proportional to $\gamma_{k}$, here we argue that different IVs may perturb the confounders ( $U$, a gene, a gene set, or multiple pathways) in slightly different ways and may lead to different CHP effects. Therefore, for an IV $k$ with a non-zero CHP effect, we decompose the effect into two components, $\alpha_{k}=\delta \gamma_{k}+\widetilde{\alpha}_{k}$. The first component $\left(\delta \gamma_{k}\right)$ is the IV-shared confounding effect, and is proportional to IV-strength, $\gamma_{k}$. The second component, $\widetilde{\alpha}_{k}$, describes how the IV-specific perturbation on confounders (i.e., the unique effect of the IV on the confounders) may affect outcome, and is independent of $\gamma_{k}$. We reparametraize our model as

$$
\Gamma_{k}= \begin{cases}\beta_{1} \gamma_{k}+\theta_{k}, & \text { if } k \in \text { IV Set } 1 \text { with no CHP } \\ \beta_{2} \gamma_{k}+\theta_{k}+\widetilde{\alpha}_{k}, & \text { if } k \in \text { IV Set } 2 \text { with CHP, }\end{cases}
$$

where $\beta_{1}$ is the causal effect of interest and can be estimated using IVs without CHP. The parameter $\beta_{2}=\beta_{1}+\delta$ is a nuisance parameter capturing both $\beta_{1}$ and $\delta$, where $\delta$ is the IV-shared confounding parameter due to CHP. MR-CUE is built on a Bayesian hierarchical model that estimates the parameters from the above model and obtains inference via Gibbs sampling.

In Fig. 1 $1 \mathrm{~b}$, we illustrate our model using a real data example to assess the causal effect of BMI on TG. When plotting IV-to-BMI effects against IV-to-TG effects in Fig. 1 b, there is a positive causal relationship for some IVs (blue) while there are a few other IVs entailing a different pattern with an opposite slope (red). The proposed MR-CUE model identifies the IVs affected by CHP (red dots), and estimates the causal effect from BMI on TG using IVs not affected by CHP (blue dots). The unconfounded causal effect is estimated to be significant and positive, $\hat{\beta}_{1(\mathrm{BMI} \rightarrow \mathrm{TG})}=0.262$. For IVs affected by CHP, their estimated causal effects is significant and negative, $\hat{\beta}_{2(\mathrm{BMI} \rightarrow \mathrm{TG})}=-0.655$, due to the large and negative confounding bias $\delta$. As further illustrated in Fig. 1k, MR-CUE reduces false positive findings due to reverse causation by identifying the IVs affected by CHP and quantifying the uncertainty in the estimation/identification. Without properly handling CHP, one may obtain a crude sum of effect estimates combining the unconfounded and the confounded effects. In the BMI-TG example, we observe that the compound effects (red), $\hat{\beta}_{2}$ 's, for both BMI-to-TG and TG-to-BMI are significant and negative, due to the shared confounding. While the unconfounded effect is only significant from BMI to TG, not the reverse. In the presence of unadjusted CHP, one 
may suffer from a reduced power or an inflated type I error rate depending on the direction of confounding effect.

In practice, there is often no clear cut for IVs unaffected or affected by CHP due to trait polygenicity and LD. The uncertainty of each variant belonging to either IV Set 1 or Set 2 can be accounted for by modeling a latent variable, $\eta_{k}$. MR-CUE imposes a spike-slab prior ${ }^{13}{ }^{14}$ for $\widetilde{\alpha}_{k}$, with a spike (mass density) at zero and a slab spreading over a wide range of plausible values. MR-CUE quantifies the the probability of each variant being affected by CHP. Different than existing methods, MR-CUE provides the flexibility for selecting IVs likely from Set I for causal effect estimation while considering selection/estimation uncertainty, and it can account for CHP effects at different levels of confidence. To further allow IVs in LD, MR-CUE partitions the whole genome into independent blocks and introduce a group latent variable, $\eta_{l}$, for IVs in same blocks (see Methods). By quantifying the uncertainty in latent IV status, MR-CUE not only provides unbiased causal effect estimates but also works as a useful tool for further examining the potential shared genetic components underlying the exposure and outcome. The IVs estimated to have CHP and their cis-associated genes may imply common genes and genetic pathways associated with both exposure and outcome.

\section{MR-CUE identifies IVs with CHP effects, estimates the causal effects and reduces false positives}

We conducted simulation studies to evaluate the performance of MR-CUE and compare with existing MR methods in a variety of scenarios. We first generated genotype matrices from different LD patterns (see Methods). Both exposure and outcome were simulated based on polygenic architecture as shown in Eqn. (15). All IVs $(p=1,000)$ contributed a total heritability of 0.1 to exposure, while the heritability for outcome can be decomposed as variation through the causal effect $\left(\beta_{1}\right)$, variation contributed by UHP $(\theta)$ and variation attributable to CHP $(\alpha)$. We controlled the combinatorial values for heritability due to UHP and CHP, denoted as $h_{\theta}^{2}$ and $h_{\alpha}^{2}$, respectively. As discussed earlier, we assumed that CHP is due to shared genetic components between exposure and outcome traits and only a proportion of IVs have nonzero CHP effects. We performed single-variant association tests to obtain the summary statistics for both IV-to-exposure and IV-to-outcome associations as input for MR analyses.

We compared MR-CUE with eight other methods, including CAUSE ${ }^{9}$, cML-MA ${ }^{10}$, MRCorr $^{215}$, MR-LDP $^{8}$, GSMR $^{3}$, RAPS $^{6}$, IVW$^{16}$, and MR-Egger ${ }^{5}$. For cML-MA, we evaluated its performance using its two default versions, i.e., cML-MA-BIC and cML-MA-BIC-DP. Among those methods, MR-LDP, RAPS, IVW, MR-Egger, and GSMR assumed that no IV/variant is affected by correlated horizontal pleiotropy, but allowed IVs to have uncorrelated horizontal pleiotropic effects. The proposed MR-CUE and two other methods, CAUSE and cML-MA, allowed IVs to have both uncorrelated and correlated horizontal pleiotropy. Among all competing methods, MR-CUE, MR-LDP, and MR-Corr ${ }^{2}$, can handle variants in moderate-to-strong LD, and two methods, CAUSE and GSMR, allowed for variants in weak LD.

First, we evaluated the performance of MR-CUE in selection/identification for IVs with CHP effects. Note that MR-CUE provided a quantitative metric for this purpose. We considered two prior distributions, i.e., the default prior (a Beta distribution with shape parameters being 2 and $L$, the number of LD blocks) and the non-informative prior, Beta(1,1). Here, we considered 
$h_{\theta}^{2}=0.02$ or $0.05, h_{\alpha}^{2}=0.05$ or 0.1 , and the correlation between $\alpha_{k}$ and $\gamma_{k}$ being $\rho_{\alpha \gamma}=0.2$. Note that when $\rho_{\alpha \gamma}=0$, only UHP is present. We also considered moderate and strong LD structure $(r=0.4,0.8)$ with autoregressive correlation. Fig. 2 a showed the false discovery rate (FDR) for identifying IVs with CHP effects and Fig. S3a (Supplementary Materials) showed the corresponding area under the curve (AUC) of the receiver operating characteristic (ROC) curve. MR-CUE with the default prior can control the FDR at the nominal level of 0.1 while achieving a high level of AUC. Next, we evaluated the performance of type I error rate control (Fig. 2p) for all competing methods. MR-CUE could control type I error rates in all settings while CAUSE was generally conservative. cML-MA-BIC suffered from inflated type I error rates while its data perturbation version, cML-MA-BIC-DP, could control the type I error rate at the expenses of power reduction (Fig. 2e). Since MR-Corr ${ }^{2}$ accounts for only CHP effects, it could control the type I error rates better than MR-LDP, which does not allow for IVs with CHP effects. Simulations for CAUSE, GSMR, RAPS, IVW and MR-Egger were all based on independent IVs after SNP clumping, since those methods were initially proposed using IVs in weak-to-moderate LD. With independent IVs, RAPS, IVW and MR-Egger could generally control the type I error rates, up to some slight inflation. We also performed simulations with a larger number of IVs $(p=2,000)$ and a stronger correlation between IV-to-exposure and CHP effects, $\rho_{\alpha \gamma}$. The results were largely similar and additional details were provided in Fig. S1 S4 (Supplementary Materials). When the correlation in CHP $\left(\rho_{\alpha \gamma}\right)$ was stronger, RAPS, IVW and MR-Egger suffered from increased levels of inflation in type I error rate.

We compared the power of each method by varying $h_{\gamma}^{2}$ (Fig. 2f) while fixing $h_{\theta}^{2}=0.1$ , $h_{\alpha}^{2}=0.05, r=0.4$, and $\rho_{\alpha \gamma}=0.2$. MR-CUE achieved the highest power among the methods that could control the type I error rates. CAUSE, as a conservative method, was under-powered 9 . Although cML-MA-BIC had a high power, its type I error rate was inflated. Its data perturbation version, cML-MA-BIC-DP, was less powerful than MR-CUE. We also considered other simulation settings with different $h_{\theta}^{2}, h_{\alpha}^{2}$, autoregressive coefficient $r$ for LD, and correlation $\rho_{\alpha \gamma}$ in CHP. Results were similar, and additional details were provided in Fig. S5 - S8 (Supplementary Materials).

Next, we evaluated whether MR-CUE could distinguish causal relationship from reverse causality. Reverse causality occurs when there exist IVs affecting the exposure and outcome traits through some shared confounding factors. Since MR-CUE is capable of identifying IVs with CHP effects, it is expected to identify the direction of true causal effect and reduce false positive findings due to reverse causality. To examine this, we simulated data with a causal effect from a trait $\mathrm{A}$ on a trait $\mathrm{B}\left(\beta_{\mathrm{A} \rightarrow \mathrm{B}} \neq 0\right)$, and tested for a reverse causal effect from $\mathrm{B}$ on $\mathrm{A}(\mathrm{B} \rightarrow \mathrm{A})$ using MR-CUE and other methods. The simulation details were provided in the Methods Section. In all scenarios, we fixed the heritability for exposure and outcome at 0.3 and 0.25 , respectively. For each simulation replicate, we applied the above MR methods for assessing the causal effects in both directions. We evaluated and compared the powers for detecting the true causal effect of exposure A on outcome B, while also compared the type I error rates for the reverse causal effect of outcome B on exposure A. Fig. $2 \mathrm{~d}$ showed the ROC curves using 100 simulated replicates at varying significance thresholds. MR-CUE, CAUSE and cML-MA-BIC-DP could distinguish causal effects from reverse causation in all simulations, while other methods cannot.

[Figure 2 about here.] 
Additionally, we evaluated the performance of MR-CUE and other methods using real data with negative and positive control ${ }^{17}$, with varying IV selection thresholds. In the analyses of negative control outcomes, we used self-reported tanning ability and hair color as outcome, since both traits were largely determined at birth and were unlikely to be affected by other traits we considered 18 . We considered 16 complex traits and diseases (Table S4, Supplementary Materials) as exposure to evaluate the control of type I error rates for MR-CUE and other MR methods. For each method, we applied five different IV selection thresholds to evaluate the sensitivity of different methods to IV selection criteria. Fig. 22 showed the quantile-quantile (QQ) plot of negative log base 10 of $p$-values for MR-CUE and other methods when IV selection threshold was $5 \times 10^{-4}$. MR-CUE and some existing MR methods including MR-LDP, RAPS, IVW and MR-Egger can well control type I error rates, with $p$-values falling within the $95 \%$ confidence band of the null distribution. Note that in the analyses of negative control outcomes, MR methods without considering CHP performed well. This was probably because that the outcomes considered were not polygenic and there was no CHP effects. On the other hand, MR-Corr ${ }^{2}$ and GSMR had inflated $p$-values while CAUSE had deflated $p$-values. MR-Corr ${ }^{2}$ had inflated type I error rates possibly because it only considered CHP but not UHP. In the analyses of positive controls, we selected 100 established pairs of traits and diseases with causal relationships supported by exiting literature. The pairs of exposure and outcome were listed in Supplementary Table S5. We also applied different IV selection thresholds to evaluate the sensitivity of results to IV selection. Fig. 2f showed the QQ plots of negative log base 10 of $p$-values using $5 \times 10^{-4}$ as the IV selection threshold. The QQ plots using other thresholds were provided in the Supplementary Fig. S9 - S10. In all scenarios, MR-CUE had the highest power. GSMR also had high powers but suffered from inflated type I error rates as shown in both simulations and negative control analyses.

\section{Examining the effects of interleukin 6 on multiple traits/diseases implies shared genes and pathways as sources of CHP}

Interleukin 6 (IL-6) is a key inflammatory cytokine, and has both pro- and anti-inflammatory properties. It plays an important role in immune-related processes and pathways $\frac{19}{19}$. Here we applied MR-CUE and other MR methods to evaluate the causal effects of IL- 6 on 27 complex traits and diseases (Table S6, Supplementary Materials). The soluble IL-6 receptor (sIL6R), a negative regulator of IL-6 signaling, has been suggested to affect many complex traits and diseases including lipid levels (e.g., high density lipoprotein cholesterol, HDL-c), both severity and susceptibility of COVID-19, heart diseases (e.g., atrial fibrillation, AF), autoimmune diseases (e.g., Crohn's disease, CD), and others ${ }^{19}[20$. We analyzed those complex traits/diseases and other diseases that may not be affected by IL-6. Supplementary Tables S1 and S9 summarized the $p$-values and the estimated causal effects for MR-CUE and other methods.

[Figure 3 about here.]

IL-6 is a multifunctional cytokine and is highly polygenic with a heritability estimate of up to $61 \% 21$. In addition to estimating the causal effects of IL-6, we further obtained the posterior probabilities of IVs having CHP effects on each of the 27 outcomes, $\operatorname{Pr}\left(\eta_{l}=1\right.$ |data $)$, 
from each chromosome clustered in blocks. In Fig. 33 right panel, we plotted the strengths of CHP effects for IVs across all chromosomes for 27 outcomes, with estimated causal effects shown in the very right column. In Fig. 33 left panel, we also plotted the genetic correlations among 27 outcome traits estimated by LDSC11. From the heatmap, we observed that traits in high genetic correlations tend to have similar or dependent estimated causal effects of IL-6, e.g., COVID19 severity and susceptibility; any stroke (AS), any ischemic stroke (AIS) and cardio-embolic stroke (CES). Those outcomes also presented similar patterns of CHP effects. On the other hand, traits in mild-to-moderate genetic correlations, e.g., bone mass density (BMD), blood urea nitrogen (BUN), major depressive disorder (MDD), bipolar disorder (BIP), and schizophrenia (SCZ), may not share causal effect estimates but could still share CHP effect patterns. CHP effects could be present when there are no causal effects.

We further identified the IVs with significant CHP effects, $\operatorname{Pr}\left(\eta_{l}=1 \mid\right.$ data $)>0.8$, and examined the genes in cis (1MB distance) and being associated with those IVs ( $p$-value $<0.05)$. The identified genes and gene sets may shed light on the shared pathways between IL-6 and the examined complex outcomes. In Fig. 3b, we plotted the heatmap of selected cis-genes associated with at least one IV affected by CHP across multiple outcomes, with color indicating the strength of the most significant association of the gene and its cis-IVs with CHP. There were many genes involved in the same pathways and being identified as IV-associated shared factors across multiple outcomes. Those shared genes may partially explain the observed genetic correlations among those 27 traits/diseases in Fig 33 (left panel). Specifically, MR-CUE identified 13 S100 genes encoding S100 proteins located in the chromosome 1q21 region. The S100 proteins belong to a family of calcium-binding cytosolic proteins and have a broad range of intracellular and extracellular functions. The extracellular S100 proteins play a crucial role in the regulation of immune homeostasis, post-traumatic injury, and inflammation ${ }^{22}$. S100 proteins trigger inflammation through their interactions with receptors for $R A G E$ and $T L R \mathbb{4}^{23}$. S100A12 has been shown to induce the production of pro-inflammatory cytokine IL-6 and IL-8 in both a dose-dependent and time-dependent manner ${ }^{22}$. Additionally, S100 proteins play a significant role to the development of chronic inflammatory and auto-inflammatory diseases 2422. MR-CUE also identified some genes in cornified envelope pathway, SPRR family and $I V L$. These genes together with $S 100$ genes constituted the epidermal differentiation complex that are essential for epidermal differentiation, building the first-line defense against external assaults and protecting our bodies from dehydration ${ }^{26}$. Genes in ATPase complex were identified to play a shared role as well. Existing literature ${ }^{27}$ reported that the overexpression of KAT5 gene potentiated transcription of downstream antiviral genes including $I L-6$. Other works ${ }^{28}$ reported that histone methyltransferase $A S H 1 L$ suppresses $T L R$-induced $I L-6$ production.

The above analysis also showed that different IVs with CHP effects may be involved in multiple pathways entailed by multiple sources of genetic confounding. The confounding effect on outcome could be IV-specific. MR-CUE allows the estimation of an overall CHP effect while accounting for IV-specific variation/perturbation to confounders and improves the estimation of CHP. By closely examining the IVs with CHP effects and their cis-associated genes, we identified genes and gene sets that were highly inter-connected as suggested sources of genetic confounding and further informed potential shared genetic etiology among the traits examined. 


\section{MR-CUE informs type 2 diabetes-related pathways for multiple risk factors across two populations}

We applied MR-CUE and other MR methods to test for causal effects of potential risk exposures for T2D in the European and East Asian populations. Type 2 diabetes (T2D) is a form of diabetes characterized by high blood sugar, insulin resistance, and relative lack of insulin ${ }^{29}$. T2D is high polygenic and has a complex etiology 30 [31. Examining multiple potential risk exposures for T2D may reveal common patterns in the etiology for related factors while also presenting unique characteristics for different types of factors. Established risk factors for T2D include both lifestyle factors, such as overweight and obesity, and medical conditions ${ }^{32}$. We also considered other exposure traits, including lipid levels, e.g., TG and high-density lipoprotein cholesterol (HDL-c), blood cell parameters, e.g., counts for red blood cells (RBC) and white blood cells (WBC), insulin-resistance-related factors, e.g., fasting insulin (FI), fasting glucose (FG) and HbA1c, and others. We examined 29 and 14 exposures for T2D in European and East Asian populations, respectively. The full list of exposure traits/diseases was provided in the Supplementary Tables S7 - S8. Tables S2 - S3 and S10 - S11 (Supplementary Materials) summarized the $p$-values and the estimated causal effects for MR-CUE and other methods. We further used MR-CUE to examine T2D-related pathways among multiple risk factors in both populations.

Some exposures for T2D are significant in both populations, such as obesity and blood cell parameters. Obesity is a well-known risk factor for T2D and the associations of blood cell parameters and T2D were also reported in many studies 33 . HbA1c was also identified by MR-CUE in both populations and its association with hypoglycemia was reported in a previous study ${ }^{36}$. Some established T2D risk factors, including insulin resistance, insulinresistance-related factors, and other obesity factors, have genetic-association summary statistics in only the European population, and thus the cross-population comparison was not presented. MR-CUE reported significant causal effects for those factors in the European population. Cross-populations analyses using summary statistics from different populations and ethnic groups still present many challenges due to the substantially varying linkage disequilibrium (LD) patterns, difficulties in data harmonization, study heterogeneity and others. Moreover, only a proportion of the causal variants and genes for complex traits/diseases might be shared across populations, and the risk exposures for a complex disease could also differ by population. MR-CUE is robust in cross-population analyses as it offers two layers of inference - it obtains the causal effect estimation using IVs not affected by genetic confounding, while also maps the underlying genes and pathways for IVs affected by confounding.

To further investigate the shared genetic pathways for the 29 and 14 traits in the European and East Asian populations, we obtained the IVs with significant CHP effects, $\operatorname{Pr}\left(\eta_{l}=1\right.$ |data $)>$ 0.8. In Fig. 价 \& b, we plotted the strengths of CHP effects for IVs across chromosomes for both European and East Asian populations, respectively. In general, exposures with higher polygenicity tend to have more IVs with CHP. We further performed pathway analysis based on those IVs using SNPnexus ${ }^{37}$ and obtained their enriched pathways, shown in Fig. 44 \& d for European and East Asian populations, respectively. The significant causal risk factors identified by MR-CUE are similar in both populations, and the enriched pathways presented some cross-population similarity as well. MR-CUE identified both metabolism and immune response 
pathways for multiple exposures and T2D in both populations. T2D itself is an inflammatory disease triggered by disordered metabolism 38 . MR-CUE identified many metabolic-related factors, including glycine, fasting glucose, and fasting insulin, having shared genetic components in metabolism pathway with T2D. Dysregulation of lipid metabolism triggers NLRP3 activation leading to obesity-induced inflammation and insulin resistance $\underline{39}, 40$. Moreover, HbA1c that is chemically linked to a sugar was used as a screening tool to detect early T2 $\mathrm{D}^{41}$. Fasting glucose and HbA1c shared many common pathways in European population (Fig. 4c) while pathways for $\mathrm{HbA1c}$ were similar in both populations. A recent work ${ }^{42}$ reported that genetic variants in glutamate cysteine ligase conferred protection against T2D, while glycine was considered

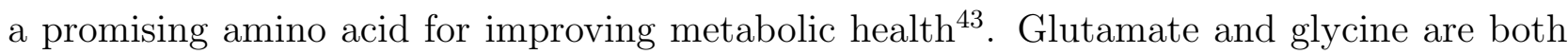
metabolites, and they play critical roles in the metabolism pathway. Glycine was reported to improve immunity and treat metabolic disorders in diabetes $\underline{44}$, while glutamate was found to be a key immunomodulator in the initiation and development of T-cell-mediated immunity 4 . We also observed that many exposures share the signal transduction pathway with T2D in both populations. Signal transduction pathway plays an important role in both red blood cell46 and $\mathrm{T} 2 \mathrm{D} 4 \sqrt[48]{4}$. Biologically, signal transduction contains insulin receptor signaling pathway that may mediate the development of T2D by endoplasmic reticulum stress $\frac{49}{\text {. }}$.

[Figure 4 about here.]

\section{Discussion}

In this work, we propose MR-CUE, a principled MR method that accounts for both uncorrelated and correlated horizontal pleiotropy. Horizontal pleiotropy arises when a genetic variant as an instrumental variable is associated with outcome not via exposure and may bias the estimation of MR inference. Correlated horizontal pleiotropy occurs when an IV is associated with unmeasured confounders, such as a shared genetic pathways underlying exposure and outcome. CHP is particularly challenging to address due to the complexity of unknown genetic pathways, yet it is frequently encountered when studying polygenic complex traits/diseases as exposure and/or outcome. MR-CUE allows all IVs to have potential uncorrelated horizontal pleiotropy, and some IVs to have CHP. MR-CUE characterizes the IVs with CHP, estimates the causal effect of exposure on outcome using the IVs not affected by CHP, and simultaneously accounts for estimation uncertainty in a Bayesian hierarchical model. MR-CUE further examines the IVs with CHP effects and their cis-associated genes for potential shared genes/pathways/mechanisms underlying exposure and outcome. With simulation studies and analyses of negative control outcomes and positive controls, we demonstrated that MR-CUE can reduce false positives due to reverse causation, control the type I error rates, and improve power and estimation precision; MR-CUE is insensitive to IV selection threshold and allow for correlated IVs; and MR-CUE identifies IVs with CHP at the desired confidence levels.

We studied the causal effects of IL-6 on multiple outcomes. By further examining the IVs with significant CHP effects and their cis-associated genes, we highlighted multiple genes that may be shared (also served as confounders) between IL-6 and some examined traits/diseases. Those suggested genes included multiple $S 100$ genes and genes in the cornified envelope pathway, shedding light on the shared genetic etiology. In another analysis, we applied MR-CUE to 
study the effects of multiple putative exposures on T2D risks in both European and East Asian populations. A cross-population analysis and comparison of multple risk exposures showed consistent causal effect estimates in both populations. We further examined the IVs with CHP effects and their enriched pathways. In both populations, it was suggested that metabolism and immune response pathways play a central role in the shared etiologies among multiple putative exposures and T2D.

MR-CUE paved the way for future cross-population MR analyses to reduce disparity. Crosspopulations MR analyses using summary statistics from different populations is still challenging due to varying LD patterns, difficulties in data harmonization, study heterogeneity and others. MR-CUE is robust in cross-population analyses as it provides double layers of inference for cross-population comparisons - it estimates the causal effect of exposure using IVs not affected by genetic confounding, while also maps the underlying genes and pathways for IVs affected by confounding.

MR-CUE has some caveats that may require further explorations. First, MR-CUE works for a single exposure and a single outcome. When the exposure is known to be highly correlated with other exposures, or when multiple outcomes may often co-occur, multi-variable MR methods accounting for both CHP and UHP may be considered. Second, MR-CUE requires multiple (at least dozens of) IVs to identify and delineate CHP effects and is not suitable for analyzing molecular risk exposures such as gene expression levels. Third, MR-CUE identifies the IVs with significant CHP effects, though the mapping of cis-associated genes/pathways from those identified IVs is still not an automated process. We are working on improving the automation of this step.

When using MR to infer causation, caution should always be exercised. By leveraging GWAS summary statistics from large genetic consortia or biobank-sized studies, MR analysis is empowered. On the other hand, insights are still limited regarding potential subgroup effects, indirect effects from different mediators between exposure and outcome, and potential exposure-mediator interactions. Further integration of MR with mediation analyses could be valuable for the development of prevention and treatment strategies towards precision medicine. 


\section{Method}

\section{MR-CUE model for independent IVs}

To estimate the causal effect in the MR-CUE model, we use the marginal effect size and standard error estimates from GWASs for exposure $(X)$ and outcome $(Y)$ diseases/traits as input. Let $\left\{\widehat{\gamma}_{k}, \widehat{\mathbf{s}}_{\gamma_{k}}\right\}$ denote the IV-to-exposure effect size and its standard error for IV $k$. Let $\left\{\widehat{\Gamma}_{k}, \widehat{S}_{\Gamma_{k}}\right\}$ denote the IV-to-outcome effect size and standard error. Let $\gamma_{k}$ and $\Gamma_{k}$ be the true marginal effect size of IV $k$ for traits $X$ and $Y$, respectively. For independent IVs, we model the distribution for the estimated effect sizes in both exposure and outcome diseases/traits using the following independently and identically distributed (i.i.d.) normal distributions,

$$
\widehat{\gamma}_{k} \sim \mathcal{N}\left(\gamma_{k}, \widehat{\mathbf{s}}_{\gamma_{k}}^{2}\right) \text {, and } \widehat{\Gamma}_{k} \sim \mathcal{N}\left(\Gamma_{k}, \widehat{\mathbf{s}}_{\Gamma_{k}}^{2}\right) .
$$

The proposed MR-CUE models the IV-to-outcome effect as a function of IV-to-exposure, and uncorrelated and correlated horizontal pleiotropic effects:

$$
\Gamma_{k}=\beta_{1} \gamma_{k}+\theta_{k}+\alpha_{k}, \quad k=1, \ldots, p,
$$

where $\theta_{k}$ and $\alpha_{k}$ capture UHP and CHP effects, respectively. The UHP effect is i.i.d. as $\theta_{k} \sim \mathcal{N}\left(0, \sigma_{\theta}^{2}\right)$. The IV-to-exposure effect $\left(\gamma_{k}\right)$ and the CHP effect $\left(\alpha_{k}\right)$ are correlated, and i.i.d. with a bivariate normal distribution:

$$
\left(\begin{array}{c}
\gamma_{k} \\
\alpha_{k}
\end{array}\right) \sim \mathcal{N}\left(\mathbf{0},\left(\begin{array}{cc}
\sigma_{\gamma}^{2}, & \rho_{\alpha \gamma} \sigma_{\gamma} \sigma_{\boldsymbol{\alpha}_{0}} \\
\rho_{\alpha \gamma} \sigma_{\gamma} \sigma_{\boldsymbol{\alpha}_{0}}, & \sigma_{\boldsymbol{\alpha}_{0}}^{2}
\end{array}\right)\right),
$$

where $\rho_{\alpha \gamma}$ is the correlation between $\gamma_{k}$ and $\alpha_{k}$.

The decomposition of CHP effects From Eqn. (4), we reparameterize $\gamma_{k}$ and $\alpha_{k}$ as follows

$$
\begin{aligned}
\gamma_{k} & \sim \mathcal{N}\left(0, \sigma_{\gamma}^{2}\right), \\
\alpha_{k} & =\rho_{\alpha \gamma} \cdot \frac{\sigma_{\alpha 0}}{\sigma_{\gamma}} \cdot \gamma_{k}+\sqrt{1-\rho_{\alpha \gamma}^{2}} \cdot \sigma_{\alpha 0} \cdot Z_{k} \stackrel{\text { def }}{=} \delta \cdot \gamma_{k}+\widetilde{\alpha}_{k},
\end{aligned}
$$

where $Z_{k}$ follows a standard normal distribution, $Z_{k} \sim \mathcal{N}(0,1)$ and $Z_{k} \Perp \gamma_{k}$, and $\delta=\rho_{\alpha \gamma} \frac{\sigma_{\alpha 0}}{\sigma_{\gamma}}$. Eqn. (5) decomposes the CHP effect $\alpha_{k}$ into two parts, with one being proportional to $\gamma_{k}$ and the other part being independent of $\gamma_{k}$, i.e., $\widetilde{\alpha}_{k} \Perp \gamma_{k}$. The decomposition in Eqn. (5) can also be viewed as a linear regression of $\alpha_{k}$ regressed on $\gamma_{k}$ with $\widetilde{\alpha}_{k}$ being the residuals. Let $\widetilde{\alpha}_{k} \sim \mathcal{N}\left(0, \sigma_{\alpha}^{2}\right)$. We call $\widetilde{\alpha}_{k}$ as the orthogonal projection of CHP. We can further parameterize the effect size of IV-to-outcome for IV $k$ as in Eqn. (2). Therefore, identifying the IVs with CHP effects in Eqn. (4) is equivalent to identifying the IVs with non-zero projected CHP, namely $\widetilde{\alpha}_{k} \neq 0$, in Eqn. (2). The estimation of causal effect $\beta_{1}$ is based on IVs with $\widetilde{\alpha}_{k}=0$.

We further introduce a latent indicator $\eta_{k}$ for each IV $k$, with $\eta_{k}=1$ for IVs with non-zero CHP effects. We impose the following spike-slab prior ${ }^{13}\left[50\right.$ on $\widetilde{\alpha}_{k}$ :

$$
\widetilde{\alpha}_{k} \sim\left\{\begin{array}{cc}
\mathcal{N}\left(0, \sigma_{\alpha}^{2}\right), & \eta_{k}=1 \\
\delta_{0}\left(\alpha_{k}\right), & \eta_{k}=0,
\end{array}\right.
$$


where $\delta_{0}$ denotes the Dirac delta function at zero, and $\eta_{k}$ follows a Bernoulli distribution with $\eta_{k} \sim \omega^{\eta_{k}}(1-\omega)^{1-\eta_{k}}$. Then, Eqn. (2) can be written as

$$
\Gamma_{k} \mid \beta_{1}, \beta_{2}, \gamma_{k}, \eta_{k}, \tau_{1}^{2}, \tau_{2}^{2} \sim\left\{\begin{array}{ll}
\mathcal{N}\left(\beta_{1} \gamma_{k}, \tau_{1}^{2}\right), & \eta_{k}=0 \\
\mathcal{N}\left(\beta_{2} \gamma_{k}, \tau_{2}^{2}\right), & \eta_{k}=1
\end{array},\right.
$$

where $\tau_{1}^{2}=\sigma_{\theta}^{2}$ and $\tau_{2}^{2}=\sigma_{\theta}^{2}+\sigma_{\alpha}^{2}$. To promote the computational efficiency in low-signal-noiseratio regime, we expand the original distribution (7) as follows $s^{51[52}$ :

$$
\Gamma_{k} \mid \beta_{1}, \beta_{2}, \gamma_{k}, \eta_{k}, \tau_{1}^{2}, \tau_{2}^{2}, \xi^{2} \sim\left\{\begin{array}{ll}
\mathcal{N}\left(\beta_{1} \gamma_{k}, \xi^{2} \tau_{1}^{2}\right), & \eta_{k}=0 \\
\mathcal{N}\left(\beta_{2} \gamma_{k}, \tau_{2}^{2}\right), & \eta_{k}=1
\end{array},\right.
$$

where $\xi^{2}$ is an expanded parameter. By combing Eqns. (3) and (8), we build the Bayesian hierarchical model with conjugate priors for hyper parameters, $\sigma_{\gamma}^{2} \sim \mathcal{I} \mathcal{G}\left(a_{\gamma}, b_{\gamma}\right), \tau_{1}^{2} \sim \mathcal{I} \mathcal{G}\left(a_{\tau 1}, b_{\tau 1}\right)$, $\tau_{2}^{2} \sim \mathcal{I} \mathcal{G}\left(a_{\tau 2}, b_{\tau 2}\right)$, and $\omega \sim \operatorname{Beta}(a, b)$.

\section{Accounting for LD}

We expand the MR-CUE model to allow for correlated IVs by modeling their LD structure. We model the estimated effect sizes in both exposure and outcome diseases/traits with approximated multivariate normal distributions $\sqrt{53}$ as follows,

$$
\begin{aligned}
\widehat{\gamma} \mid \boldsymbol{\gamma}, \widehat{\mathbf{R}}, \widehat{\mathbf{S}}_{\gamma} \sim \mathcal{N}\left(\widehat{\mathbf{S}}_{\gamma} \widehat{\mathbf{R}} \widehat{\mathbf{S}}_{\gamma}^{-1} \boldsymbol{\gamma}, \widehat{\mathbf{S}}_{\gamma} \widehat{\mathbf{R}} \widehat{\mathbf{S}}_{\gamma}\right), \\
\widehat{\boldsymbol{\Gamma}} \mid \boldsymbol{\Gamma}, \widehat{\mathbf{R}}, \widehat{\mathbf{S}}_{\Gamma} \sim \mathcal{N}\left(\widehat{\mathbf{S}}_{\Gamma} \widehat{\mathbf{R}} \widehat{\mathbf{S}}_{\Gamma}^{-1} \boldsymbol{\Gamma}, \widehat{\mathbf{S}}_{\Gamma} \widehat{\mathbf{R}} \widehat{\mathbf{S}}_{\Gamma}\right),
\end{aligned}
$$

where $\widehat{\gamma}=\left[\widehat{\gamma}_{1}, \ldots, \widehat{\gamma}_{p}\right]^{T}$ and $\widehat{\boldsymbol{\Gamma}}=\left[\widehat{\Gamma}_{1}, \ldots, \widehat{\Gamma}_{p}\right]^{T}$ are vectors for the marginal effect sizes in exposure and outcome diseases/traits, respectively; $\widehat{\mathbf{S}}_{\gamma}=\operatorname{diag}\left(\left[\widehat{\mathbf{s}}_{\gamma_{1}}, \cdots, \widehat{\mathbf{s}}_{\gamma_{p}}\right]\right)$ and $\widehat{\mathbf{S}}_{\Gamma}=$ $\operatorname{diag}\left(\left[\widehat{\mathbf{s}}_{\Gamma_{1}}, \cdots, \widehat{\mathbf{s}}_{\Gamma_{p}}\right]\right)$ are the corresponding diagonal matrices for standard errors; and $\widehat{\mathbf{R}} \in \mathbb{R}^{p \times p}$ is the estimated correlation matrix among all selected IVs. In the approximated distributions in Eqn. (9), all quantities except for $\widehat{\mathbf{R}}$ can be obtained from summary-level GWAS results while $\widehat{\mathbf{R}}$ is estimated using an independent reference panel data.

\section{Estimating LD matrix from a reference panel}

To estimate the LD matrix, we used independent reference panel data from the following sources: UK10K Project (Avon Longitudinal Study of Parents and Children, ALSPAC ${ }^{54}$, and TwinsUK ${ }^{(55)}$ ) merged with European-ancestry samples in 1000 Genome Project Phase $3^{\sqrt[56]{6}}$. There are 4,284 individuals in total. We conducted strict quality control for the reference data using PLINK $^{57}$ and GCTA ${ }^{58}$. We removed the individuals with genotype missing rates greater than $5 \%$, and further removed one pair of individuals that have genetic relatedness larger than 0.05. Since both ALSPAC and TwinsUK cohorts contain non-European samples, we further performed the principal components analysis $(\mathrm{PCA})^{59}$ followed by the analysis of hierarchical clustering on principal components $(\mathrm{HCPC})^{60}$ to extract and restrict the analysis to samples from European ancestries. After data pre-processing, roughly 3,700 samples were retained as the reference panel data.

Often it is useful to define approximately independent LD blocks a priori. Here we used LDetect ${ }^{61}$ based on an efficient signal processing approach for choosing segment boundaries between 
blocks. Consequently, LDetect partitioned the entire genome into 1,703 and 1,445 independent blocks for European and Asian populations, respectively (http://bitbucket.org/nygcresearch/ldetectdata). For each LD block, we calculated the empirical correlation matrix and further applied a simple shrinkage correlation estimator ${ }^{62}$ to obtain

$$
\widehat{\mathbf{R}}^{(l)}=\lambda \widehat{\mathbf{R}}_{\mathrm{emp}}^{(l)}+(1-\lambda) \widehat{\mathbf{I}}^{(l)},
$$

where $\widehat{\mathbf{R}}_{\mathrm{emp}}^{(l)} \in \mathbb{R}^{p_{l} \times p_{l}}$ was the empirical correlation matrix for the $l$-th block in the panel data and $\lambda \geq 0$ was a shrinkage parameter. By obtaining all $\widehat{\mathbf{R}}^{(l)} \mathrm{s}, l=1, \ldots, L$, we could further obtain $\widehat{\mathbf{R}}=\operatorname{diag}\left(\widehat{\mathbf{R}}^{(l)}\right) \in \mathbb{R}^{p \times p}$ with $\sum_{l=1}^{L} p_{l}=p$. Here we fixed the shrinkage parameter $\lambda$ at $0.85^{8}$.

A group spike-slab prior For IVs in moderate-to-strong LD, if there is a single variant $k$ with a nonzero CHP effect, the CHP effect for other nearby variants in the block would be also nonzero. In our analyses, genetic variants across the genome can be partitioned into independent blocks. IVs from different blocks could be roughly taken as independent. Thus, the projected $\widetilde{\alpha}_{k}$ is estimated in a group manner. We introduce a group-level latent status $\eta_{l}$, indicating whether IVs within the $l$-th block having nonzero CHP effects and assigning a group-level spike-slab prior as follows:

$$
\widetilde{\alpha}_{l k} \sim \begin{cases}\mathcal{N}\left(0, \sigma_{\alpha}^{2}\right), & \eta_{l}=1 \\ \delta_{0}\left(\alpha_{l k}\right), & \eta_{l}=0,\end{cases}
$$

where $\eta_{l}=1$ implies the IVs within the $l$-th block having nonzero projected CHP effects and $\eta_{l}=0$ means the projected CHP effects being all zero for IVs in the block. Here, $\eta_{l}$ is a Bernoulli random variable with probability $\omega$ being $1, \eta_{l} \sim \omega^{\eta_{l}}(1-\omega)^{1-\eta_{l}}$.

Considering IVs in LD, we have the following mixture distribution for $\Gamma_{l k}$ that is similar to Eqn. (8):

$$
\Gamma_{l k} \mid \beta_{1}, \beta_{2}, \gamma_{l k}, \eta_{l}, \tau_{1}^{2}, \tau_{2}^{2}, \xi^{2} \sim \begin{cases}\mathcal{N}\left(\beta_{1} \gamma_{l k}, \xi^{2} \tau_{1}^{2}\right), & \text { if } \eta_{l}=0 \\ \mathcal{N}\left(\beta_{2} \gamma_{l k}, \tau_{2}^{2}\right), & \text { if } \eta_{l}=1\end{cases}
$$

\section{Accounting for sample overlap}

When IV-to-exposure and IV-to-outcome summary statistics are taken from biobank-sized or consortia-based GWASs with potential overlapping samples, we need to account for the potential additional correlations. To allow overlapping samples in GWAS for both diseases/traits, we could rewrite the distribution for summary statistics in Eqn. (9) as the following matrix normal distribution for $Z$-score

$$
\widehat{\mathbf{z}}=\left[\widehat{\mathbf{z}}_{\gamma}, \widehat{\mathbf{z}}_{\boldsymbol{\Gamma}}\right] \sim \mathcal{M N}\left(\left[\widehat{\mathbf{R}} \widehat{\mathbf{S}}_{\gamma}^{-1} \boldsymbol{\gamma}, \widehat{\mathbf{R}} \widehat{\mathbf{S}}_{\boldsymbol{\Gamma}}^{-1} \boldsymbol{\Gamma}\right], \widehat{\mathbf{R}}, \mathbf{R}_{e}\right)
$$

where $\widehat{\mathbf{z}}_{\boldsymbol{\gamma}}=\widehat{\mathbf{S}}_{\gamma}^{-1} \widehat{\boldsymbol{\gamma}}, \quad \widehat{\mathbf{z}}_{\boldsymbol{\Gamma}}=\widehat{\mathbf{S}}_{\boldsymbol{\Gamma}}^{-1} \widehat{\boldsymbol{\Gamma}}$, and $\mathbf{R}_{e}=\left[\begin{array}{cc}1 & \rho_{e} \\ \rho_{e} & 1\end{array}\right]$ is the correlation matrix that accounts for sample overlap. Here, the correlation due to sample overlap $\rho_{e}$ can be estimated using summary statistics among independent variants with no associations to both exposure and outcome diseases/traits. 
We propose the following Bayesian hierarchical model for correlated IVs with overlapping samples,

$$
\begin{gathered}
\widehat{\mathbf{z}}=\left[\widehat{\mathbf{z}}_{\gamma}, \widehat{\mathbf{z}}_{\boldsymbol{\Gamma}}\right] \sim \mathcal{M N}\left(\left[\widehat{\mathbf{R}}_{\boldsymbol{\gamma}}^{-1} \boldsymbol{\gamma}, \widehat{\mathbf{R}}_{\mathbf{\Gamma}}^{-1} \boldsymbol{\Gamma}\right], \widehat{\mathbf{R}}, \widehat{\mathbf{R}}_{e}\right), \\
\Gamma_{l k} \mid \beta_{1}, \beta_{2}, \gamma_{l k}, \eta_{l}, \tau_{1}^{2}, \tau_{2}^{2}, \xi^{2} \stackrel{i i d}{\sim}\left\{\mathcal{N}\left(\beta_{1} \gamma_{l k}, \xi^{2} \tau_{1}^{2}\right)\right\}^{\left(1-\eta_{l}\right)}\left\{\mathcal{N}\left(\beta_{2} \gamma_{l k}, \tau_{2}^{2}\right)\right\}^{\eta_{l}}, \\
\gamma_{l k}\left|\sigma_{\gamma}^{2} \stackrel{i i d}{\sim} \mathcal{N}\left(0, \sigma_{\gamma}^{2}\right), \quad \widetilde{\alpha}_{l k}\right| \sigma_{\alpha}^{2} \stackrel{i i d}{\sim} \mathcal{N}\left(0, \sigma_{\alpha}^{2}\right), \quad \eta_{l} \mid \omega \stackrel{i i d}{\sim} \omega^{\eta_{l}}(1-\omega)^{1-\eta_{l}}, \\
\sigma_{\gamma}^{2} \sim \mathcal{I} \mathcal{G}\left(a_{\gamma}, b_{\gamma}\right), \quad \tau_{1}^{2} \sim \mathcal{I} \mathcal{G}\left(a_{\tau 1}, b_{\tau 1}\right), \quad \tau_{2}^{2} \sim \mathcal{I} \mathcal{G}\left(a_{\tau 2}, b_{\tau 2}\right), \\
\operatorname{Pr}\left(\xi^{2}\right) \propto \frac{1}{\xi^{2}}, \quad \eta_{l} \mid \omega \stackrel{i i d}{\sim} \omega^{\eta_{l}}(1-\omega)^{1-\eta_{l}}, \quad \omega \sim \operatorname{Beta}(a, b) .
\end{gathered}
$$

Since the estimated LD matrix is block-diagonal, the resulting Gibbs sampler can be performed in a parallel manner for each block. The algorithmic details are given in the Supplementary Materials.

\section{Generation of summary statistics in the simulation studies}

We generated the summary statistics using simulated individual-level data. We first simulated genotypes $\mathbf{G}_{x} \in \mathbb{R}^{n_{x} \times p}, \mathbf{G}_{y} \in \mathbb{R}^{n_{y} \times p}$ and $\mathbf{G}_{r} \in \mathbb{R}^{n_{r} \times p}$ for both exposure and outcome as well as for an independent reference data, respectively, where $n_{x}, n_{y}$, and $n_{r}$ were the corresponding sample sizes and $p$ was the total number of IVs. We set the number of blocks $L$ to be 100 or 200, and the number of IVs within a block to be 10, respectively. Correspondingly, the number of IVs was either 1,000 or 2,000. For all simulations, we considered $n_{x}=50,000, n_{y}=50,000$ and $n_{r}=4,000$.

We then generated a data matrix from a multivariate normal distribution $\mathcal{N}(\mathbf{0}, \boldsymbol{\Sigma}(r))$, where $r \in\{0.4,0.8\}$ represented the autoregressive correlation among IVs. We simulated genotype matrix by categorizing data matrices into dosage values $\{0,1,2\}$ according to minor allele frequency that is uniformly distributed in $[0.05,0.5]$. We then considered the following structural model to generate individual-level data

$$
\mathbf{x}=\mathbf{G}_{x} \boldsymbol{\gamma}+\mathbf{U}_{x} \boldsymbol{\psi}_{x}+\boldsymbol{\epsilon}_{x}, \quad \mathbf{y}=\beta_{1} \mathbf{x}+\mathbf{G}_{y} \boldsymbol{\alpha}+\mathbf{G}_{y} \boldsymbol{\theta}+\mathbf{U}_{y} \boldsymbol{\psi}_{y}+\boldsymbol{\epsilon}_{y}
$$

where $\mathbf{U}_{x} \in \mathbb{R}^{n_{x} \times q}$ and $\mathbf{U}_{y} \in \mathbb{R}^{n_{y} \times q}$ are the matrices for $q$ confounders for exposure and outcome, respectively; $\boldsymbol{\psi}_{x} \in \mathbb{R}^{q \times 1}$ and $\boldsymbol{\psi}_{y} \in \mathbb{R}^{q \times 1}$ are the corresponding vector of coefficients; $\boldsymbol{\epsilon}_{x} \in \mathbb{R}^{n_{x} \times 1}$ and $\boldsymbol{\epsilon}_{y} \in \mathbb{R}^{n_{y} \times 1}$ are the random errors; and $\beta_{1}$ is the causal effect of interest. In all simulations, we considered $q=50$ and each column of $\mathbf{U}_{x}$ and $\mathbf{U}_{y}$ was sampled from a standard normal distribution. The coefficients of these confounders, $\boldsymbol{\psi}_{x}$ and $\boldsymbol{\psi}_{y}$, were sampled from a bivariate normal distribution $\mathcal{N}\left(\mathbf{0}, \boldsymbol{\Sigma}_{\psi}\right)$, where $\boldsymbol{\Sigma}_{\psi}$ was a two-by-two matrix with diagonal elements of 1 and off-diagonal elements of 0.8. For CHP effects, we assumed $\gamma_{k}$ and $\alpha_{k}$ following a bivariate normal distribution $\mathcal{N}\left(\mathbf{0}, \boldsymbol{\Sigma}\left(\rho_{\alpha \gamma}\right)\right)$. We considered $\alpha_{k}$ to be sparse, i.e., only $10 \%$ of $\alpha_{k}$ was sampled from the bivariate normal distribution and the others were zero. For UHP, we assumed $\theta_{k}$ to be dense and follow an independent normal distribution, $\mathcal{N}\left(0, \sigma_{\theta}^{2}\right)$.

We further performed the single-variant analysis to obtain summary statistics, $\left\{\widehat{\gamma}_{k}, \widehat{\mathbf{s}}_{\gamma_{k}}\right\}$ and $\left\{\widehat{\Gamma}_{k}, \widehat{\mathbf{s}}_{\Gamma_{k}}\right\}, \forall k=1, \ldots, p$, for both exposure and outcome, respectively. In the simulation study, we controlled the magnitudes for $\boldsymbol{\gamma}, \boldsymbol{\alpha}$ and $\boldsymbol{\theta}$ using $h_{\gamma}^{2}=\frac{\operatorname{var}\left(\beta_{1} \mathbf{G}_{1} \boldsymbol{\gamma}\right)}{\operatorname{var}(\mathbf{y})}, h_{\alpha}^{2}=\frac{\operatorname{var}\left(\mathbf{G}_{2} \boldsymbol{\alpha}\right)}{\operatorname{var}(\mathbf{y})}$ and $h_{\theta}^{2}=\frac{\operatorname{var}\left(\mathbf{G}_{2} \boldsymbol{\theta}\right)}{\operatorname{var}(\mathbf{y})}$, respectively. We considered $h_{\gamma}^{2}=0.1$ and varied $h_{\theta}^{2} \in\{0.02,0.05\}$ and 
$h_{\alpha}^{2} \in\{0.05,0.1\}$ to evaluate the performance of MR-CUE in selecting/identifying IVs with CHP effects and in the control of type I error rates. To further examine the power, we varied $h_{\gamma}^{2}$ in a sequence of values from 0 to 0.1 while fixing other parameters.

\section{Generation of summary statistics for reverse causation analysis}

We considered the following structural model to generate individual-level data that is similar to existing work ${ }^{9}$ :

$$
\mathbf{x}=\mathbf{G}_{1} \boldsymbol{\gamma}+\mathbf{e}_{1}, \quad \mathbf{y}=\beta_{1} \mathbf{x}+\mathbf{G}_{2} \boldsymbol{\theta}+\mathbf{e}_{2},
$$

where $\boldsymbol{\gamma}$ and $\boldsymbol{\theta}$ are from two independent normal distributions. In this simulation, we first controlled the heritability of exposure and outcome, denoted as $h_{x}^{2}$ and $h_{y}^{2}$, respectively. We further assumed that $20 \%$ of the outcome heritability, $h_{y}^{2}$, can be explained by the causal effect $\left(\beta_{1}\right)$ of exposure on outcome. Thus, we have three quantities below

$$
h_{x}^{2} \stackrel{\text { def }}{=} \frac{\operatorname{var}\left(\mathbf{G}_{1} \boldsymbol{\gamma}\right)}{\operatorname{var}(\mathbf{x})}, \quad h_{y}^{2} \stackrel{\text { def }}{=} \frac{\operatorname{var}\left(\beta_{1} \mathbf{G}_{2} \boldsymbol{\gamma}+\mathbf{G}_{2} \boldsymbol{\theta}\right)}{\operatorname{var}(\mathbf{y})} \quad \text { and } \quad \frac{\operatorname{var}\left(\beta_{1} \mathbf{G}_{2} \boldsymbol{\gamma}\right)}{\operatorname{var}(\mathbf{y})}=\frac{h_{y}^{2}}{5} .
$$

We set $h_{y}^{2}=0.25, h_{x}^{2}=0.3$, and only $5 \%$ of $\gamma$ being nonzero. We fixed $r=0.4, p=2000$, and $\rho_{\alpha \gamma}=0.2$. To examine reverse causality, we applied MR-CUE and other methods to assess the causal effects in both directions for 100 simulated replicates. By varying significance thresholds, we obtained the ROC curves for true positives vs. false positives averaged over the 100 replicates. 


\section{References}

${ }^{1}$ Smith, G. D. and Ebrahim, S. (2004). Mendelian randomization: prospects, potentials, and limitations. International journal of epidemiology 33, 30-42.

${ }^{2}$ Ference, B. A., Yoo, W., Alesh, I., Mahajan, N., Mirowska, K. K., Mewada, A., Kahn, J., Afonso, L., Williams, K. A., and Flack, J. M. (2012). Effect of long-term exposure to lower low-density lipoprotein cholesterol beginning early in life on the risk of coronary heart disease: a mendelian randomization analysis. Journal of the American College of Cardiology 60, 2631-2639.

${ }^{3}$ Zhu, Z., Zheng, Z., Zhang, F., Wu, Y., Trzaskowski, M., Maier, R., Robinson, M. R., McGrath, J. J., Visscher, P. M., Wray, N. R., et al. (2018). Causal associations between risk factors and common diseases inferred from gwas summary data. Nature communications 9, 1-12.

${ }^{4}$ Verbanck, M., Chen, C.-y., Neale, B., and Do, R. (2018). Detection of widespread horizontal pleiotropy in causal relationships inferred from mendelian randomization between complex traits and diseases. Nature genetics 50, 693-698.

${ }^{5}$ Bowden, J., Davey Smith, G., and Burgess, S. (2015). Mendelian randomization with invalid instruments: effect estimation and bias detection through egger regression. International journal of epidemiology $44,512-525$.

${ }^{6}$ Zhao, Q., Wang, J., Hemani, G., Bowden, J., Small, D. S., et al. (2020). Statistical inference in two-sample summary-data mendelian randomization using robust adjusted profile score. Annals of Statistics 48, 1742-1769.

${ }^{7}$ Zhao, J., Ming, J., Hu, X., Chen, G., Liu, J., and Yang, C. (2020). Bayesian weighted mendelian randomization for causal inference based on summary statistics. Bioinformatics $36,1501-1508$.

${ }^{8}$ Cheng, Q., Yang, Y., Shi, X., Yeung, K.-F., Yang, C., Peng, H., and Liu, J. (2020). Mr-ldp: a two-sample mendelian randomization for gwas summary statistics accounting for linkage disequilibrium and horizontal pleiotropy. NAR Genomics and Bioinformatics 2, lqaa028.

${ }^{9}$ Morrison, J., Knoblauch, N., Marcus, J. H., Stephens, M., and He, X. (2020). Mendelian randomization accounting for correlated and uncorrelated pleiotropic effects using genomewide summary statistics. Nature genetics 52, 740-747.

${ }^{10}$ Xue, H., Shen, X., and Pan, W. (2021). Constrained maximum likelihood-based mendelian randomization robust to both correlated and uncorrelated pleiotropic effects. The American Journal of Human Genetics 108, 1251-1269.

${ }^{11}$ Bulik-Sullivan, B., Finucane, H. K., Anttila, V., Gusev, A., Day, F. R., Loh, P.-R., Duncan, L., Perry, J. R., Patterson, N., Robinson, E. B., et al. (2015). An atlas of genetic correlations across human diseases and traits. Nature genetics 47, 1236-1241. 
${ }^{12}$ Van Rheenen, W., Peyrot, W. J., Schork, A. J., Lee, S. H., and Wray, N. R. (2019). Genetic correlations of polygenic disease traits: from theory to practice. Nature Reviews Genetics 20, $567-581$.

${ }^{13}$ Ishwaran, H., Rao, J. S., et al. (2005). Spike and slab variable selection: frequentist and bayesian strategies. The Annals of Statistics 33, 730-773.

${ }^{14}$ Malsiner-Walli, G. and Wagner, H. (2018). Comparing spike and slab priors for bayesian variable selection. arXiv preprint arXiv:1812.07259.

${ }^{15}$ Cheng, Q., Sun, B., Xia, Y., and Liu, J. (2020). Accounting for correlated horizontal pleiotropy in two-sample mendelian randomization using correlated instrumental variants. arXiv preprint arXiv:2009.00399.

${ }^{16}$ Burgess, S., Butterworth, A., and Thompson, S. (2013). Mendelian randomization analysis with multiple genetic variants using summarized data. Genet Epidemiol 37(7), 658-665.

${ }^{17}$ Burgess, S., Smith, G. D., Davies, N. M., Dudbridge, F., Gill, D., Glymour, M. M., Hartwig, F. P., Holmes, M. V., Minelli, C., Relton, C. L., et al. (2019). Guidelines for performing mendelian randomization investigations. Wellcome Open Research 4.

${ }^{18}$ Sanderson, E., Richardson, T., Hemani, G., and Smith, G. D. (2020). The use of negative control outcomes in mendelian randomisation to detect potential population stratification or selection bias. BioRxiv.

19 Tanaka, T., Narazaki, M., and Kishimoto, T. (2014). Il-6 in inflammation, immunity, and disease. Cold Spring Harbor perspectives in biology 6, a016295.

${ }^{20}$ McElvaney, O. J., Curley, G. F., Rose-John, S., and McElvaney, N. G. (2021). Interleukin-6: obstacles to targeting a complex cytokine in critical illness. The Lancet Respiratory Medicine.

${ }^{21}$ Ahluwalia, T. S., Prins, B. P., Abdollahi, M., Armstrong, N. J., Aslibekyan, S., Bain, L., Jefferis, B., Baumert, J., Beekman, M., Ben-Shlomo, Y., et al. (2021). Genome-wide association study of circulating interleukin 6 levels identifies novel loci. Human molecular genetics 30, 393-409.

${ }^{22}$ Xia, C., Braunstein, Z., Toomey, A. C., Zhong, J., and Rao, X. (2018). S100 proteins as an important regulator of macrophage inflammation. Frontiers in immunology 8, 1908.

${ }^{23}$ Vogl, T., Tenbrock, K., Ludwig, S., Leukert, N., Ehrhardt, C., Van Zoelen, M. A., Nacken, W., Foell, D., Van der Poll, T., Sorg, C., et al. (2007). Mrp8 and mrp14 are endogenous activators of toll-like receptor 4 , promoting lethal, endotoxin-induced shock. Nature medicine 13, 1042-1049.

${ }^{24}$ Perera, C., McNeil, H. P., and Geczy, C. L. (2010). S100 calgranulins in inflammatory arthritis. Immunology and cell biology 88, 41-49.

${ }^{25}$ Heizmann, C. W. (2002). The multifunctional s100 protein family. Calcium-Binding Protein Protocols pp. 69-80. 
${ }^{26}$ Kypriotou, M., Huber, M., and Hohl, D. (2012). The human epidermal differentiation complex: cornified envelope precursors, s100 proteins and the 'fused genes' family. Experimental dermatology 21, 643-649.

${ }^{27}$ Song, Z.-M., Lin, H., Yi, X.-M., Guo, W., Hu, M.-M., and Shu, H.-B. (2020). Kat5 acetylates cgas to promote innate immune response to dna virus. Proceedings of the National Academy of Sciences 117, 21568-21575.

${ }^{28}$ Xia, M., Liu, J., Wu, X., Liu, S., Li, G., Han, C., Song, L., Li, Z., Wang, Q., Wang, J., et al. (2013). Histone methyltransferase ash1l suppresses interleukin-6 production and inflammatory autoimmune diseases by inducing the ubiquitin-editing enzyme a20. Immunity 39, 470-481.

${ }^{29}$ Symptoms \& Causes of Diabetes. https://www.niddk.nih.gov/health-information/ diabetes/overview/symptoms-causes?dkrd=hispt0015. Accessed: 2016-02-10.

${ }^{30}$ Langenberg, C. and Lotta, L. A. (2018). Genomic insights into the causes of type 2 diabetes. The Lancet 391, 2463-2474.

${ }^{31}$ Xue, A., Wu, Y., Zhu, Z., Zhang, F., Kemper, K. E., Zheng, Z., Yengo, L., Lloyd-Jones, L. R., Sidorenko, J., Wu, Y., et al. (2018). Genome-wide association analyses identify 143 risk variants and putative regulatory mechanisms for type 2 diabetes. Nature communications 9 , $1-14$.

${ }^{32}$ Funnell, M. M. and Anderson, R. M. (2008). Influencing self-management: from compliance to collaboration. In Type 2 Diabetes Mellitus In Type 2 Diabetes Mellitus. (Springer).

33 Tong, P. C., Lee, K.-F., So, W.-Y., Ng, M. H., Chan, W.-B., Lo, M. K., Chan, N. N., and Chan, J. C. (2004). White blood cell count is associated with macro-and microvascular complications in chinese patients with type 2 diabetes. Diabetes care 27, 216-222.

${ }^{34}$ Demirtunc, R., Duman, D., Basar, M., Bilgi, M., Teomete, M., and Garip, T. (2009). The relationship between glycemic control and platelet activity in type 2 diabetes mellitus. Journal of Diabetes and its Complications 23, 89-94.

${ }^{35}$ Magri, C. J. and Fava, S. (2014). Red blood cell distribution width and diabetes-associated complications. Diabetes \& Metabolic Syndrome: Clinical Research \& Reviews 8, 13-17.

${ }^{36}$ Lipska, K. J., Warton, E. M., Huang, E. S., Moffet, H. H., Inzucchi, S. E., Krumholz, H. M., and Karter, A. J. (2013). Hba1c and risk of severe hypoglycemia in type 2 diabetes: the diabetes and aging study. Diabetes care 36, 3535-3542.

${ }^{37}$ Oscanoa, J., Sivapalan, L., Gadaleta, E., Dayem Ullah, A. Z., Lemoine, N. R., and Chelala, C. (2020). Snpnexus: a web server for functional annotation of human genome sequence variation (2020 update). Nucleic acids research 48, W185-W192.

${ }^{38}$ Donath, M. Y. and Shoelson, S. E. (2011). Type 2 diabetes as an inflammatory disease. Nature reviews immunology 11, 98-107. 
${ }^{39}$ Vandanmagsar, B., Youm, Y.-H., Ravussin, A., Galgani, J. E., Stadler, K., Mynatt, R. L., Ravussin, E., Stephens, J. M., and Dixit, V. D. (2011). The nlrp3 inflammasome instigates obesity-induced inflammation and insulin resistance. Nature medicine 17, 179-188.

${ }^{40}$ Hameed, I., Masoodi, S. R., Mir, S. A., Nabi, M., Ghazanfar, K., and Ganai, B. A. (2015). Type 2 diabetes mellitus: from a metabolic disorder to an inflammatory condition. World journal of diabetes 6,598 .

${ }^{41}$ Bennett, C., Guo, M., and Dharmage, S. (2007). Hba1c as a screening tool for detection of type 2 diabetes: a systematic review. Diabetic medicine 24, 333-343.

${ }^{42}$ Azarova, I., Klyosova, E., Lazarenko, V., Konoplya, A., and Polonikov, A. (2020). Genetic variants in glutamate cysteine ligase confer protection against type 2 diabetes. Molecular Biology Reports 47, 5793-5805.

43 Alves, A., Bassot, A., Bulteau, A.-L., Pirola, L., and Morio, B. (2019). Glycine metabolism and its alterations in obesity and metabolic diseases. Nutrients 11, 1356.

${ }^{44}$ Wang, W., Wu, Z., Dai, Z., Yang, Y., Wang, J., and Wu, G. (2013). Glycine metabolism in animals and humans: implications for nutrition and health. Amino acids 45, 463-477.

${ }^{45}$ Pacheco, R., Gallart, T., Lluis, C., and Franco, R. (2007). Role of glutamate on t-cell mediated immunity. Journal of neuroimmunology 185, 9-19.

${ }^{46}$ Richmond, T. D., Chohan, M., and Barber, D. L. (2005). Turning cells red: signal transduction mediated by erythropoietin. Trends in cell biology 15, 146-155.

${ }^{47}$ Mandrup-Poulsen, T. (2003). Apoptotic signal transduction pathways in diabetes. Biochemical pharmacology 66, 1433-1440.

${ }^{48}$ Björnholm, M. and Zierath, J. (2005). Insulin signal transduction in human skeletal muscle: identifying the defects in type ii diabetes. Biochemical Society Transactions 33, 354-357.

${ }^{49}$ Özcan, U., Cao, Q., Yilmaz, E., Lee, A.-H., Iwakoshi, N. N., Özdelen, E., Tuncman, G., Görgün, C., Glimcher, L. H., and Hotamisligil, G. S. (2004). Endoplasmic reticulum stress links obesity, insulin action, and type 2 diabetes. Science 306, 457-461.

${ }^{50}$ Shi, X., Jiao, Y., Yang, Y., Cheng, C.-Y., Yang, C., Lin, X., and Liu, J. (2019). Vimco: variational inference for multiple correlated outcomes in genome-wide association studies. Bioinformatics 35, 3693-3700.

${ }^{51}$ Gelman, A., Carlin, J. B., Stern, H. S., Dunson, D. B., Vehtari, A., and Rubin, D. B. (2013). Bayesian data analysis. (CRC press).

${ }^{52}$ Gelman, A. et al. (2006). Prior distributions for variance parameters in hierarchical models (comment on article by browne and draper). Bayesian analysis 1, 515-534.

${ }^{53}$ Zhu, X. and Stephens, M. (2017). Bayesian large-scale multiple regression with summary statistics from genome-wide association studies. The annals of applied statistics 11, 1561. 
${ }^{54}$ Boyd, A., Thomas, R., Hansell, A. L., Gulliver, J., Hicks, L. M., Griggs, R., Vande Hey, J., Taylor, C. M., Morris, T., Golding, J., et al. (2019). Data resource profile: The alspac birth cohort as a platform to study the relationship of environment and health and social factors. International journal of epidemiology 48, 1038-1039k.

${ }^{55}$ Moayyeri, A., Hammond, C. J., Valdes, A. M., and Spector, T. D. (2013). Cohort profile: Twinsuk and healthy ageing twin study. International journal of epidemiology 42, 76-85.

${ }^{56}$ Fairley, S., Lowy-Gallego, E., Perry, E., and Flicek, P. (2020). The international genome sample resource (igsr) collection of open human genomic variation resources. Nucleic Acids Research 48, D941-D947.

${ }^{57}$ Purcell, S., Neale, B., Todd-Brown, K., Thomas, L., Ferreira, M. A., Bender, D., Maller, J., Sklar, P., De Bakker, P. I., Daly, M. J., et al. (2007). Plink: a tool set for whole-genome association and population-based linkage analyses. The American journal of human genetics $81,559-575$.

${ }^{58}$ Yang, J., Lee, S. H., Goddard, M. E., and Visscher, P. M. (2011). GCTA: a tool for genome-wide complex trait analysis. The American Journal of Human Genetics 88, 76-82.

59 Turner, S., Armstrong, L. L., Bradford, Y., Carlson, C. S., Crawford, D. C., Crenshaw, A. T., De Andrade, M., Doheny, K. F., Haines, J. L., Hayes, G., et al. (2011). Quality control procedures for genome-wide association studies. Current protocols in human genetics 68, $1-19$.

${ }^{60}$ Husson, F., Josse, J., and Pages, J. (2010). Principal component methods-hierarchical clustering-partitional clustering: why would we need to choose for visualizing data. Applied Mathematics Department pp. 1-17.

${ }^{61}$ Berisa, T. and Pickrell, J. K. (2016). Approximately independent linkage disequilibrium blocks in human populations. Bioinformatics 32, 283.

${ }^{62}$ Schäfer, J. and Strimmer, K. (2005). A shrinkage approach to large-scale covariance matrix estimation and implications for functional genomics. Statistical applications in genetics and molecular biology 4 . 


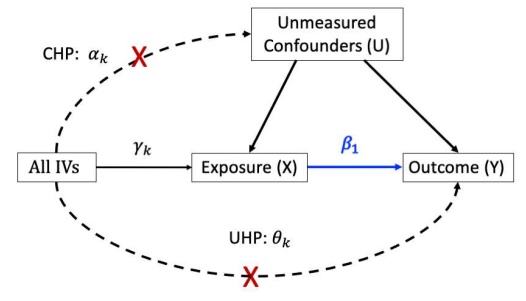

(a)

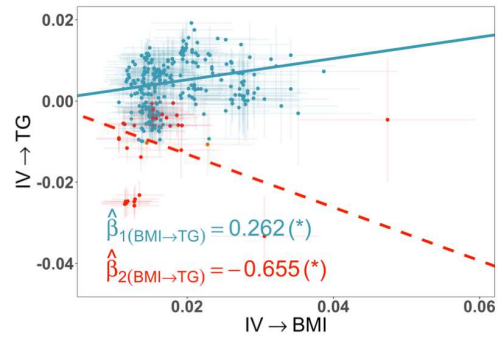

(b)

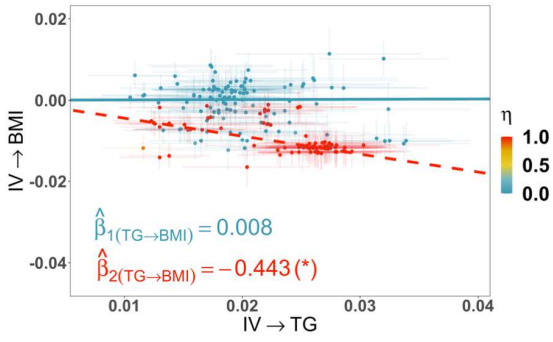

(c)
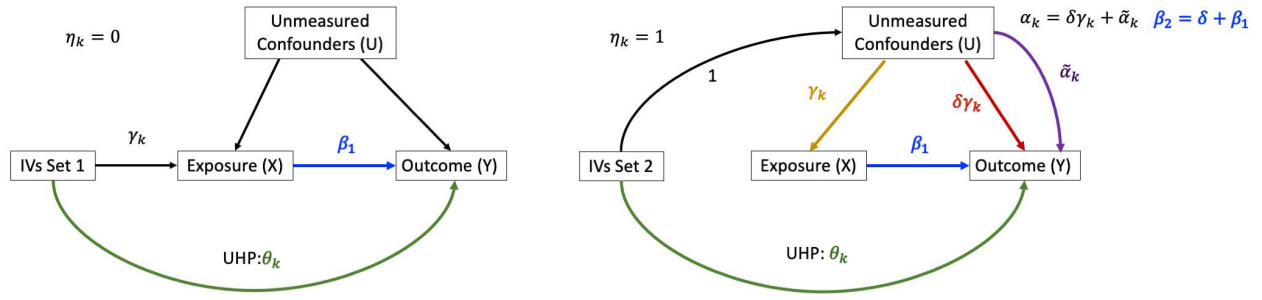

(d)

Figure 1: (a) The causal diagram by classic MR methods. Classic MR methods assume IV to affect outcome through only exposure. (b) An illustrative example of estimating the causal effect of BMI on TG in the presence of CHP. (c) The reverse causation estimation of TG on BMI confounded by CHP. When estimating the effect of BMI on TG, some IVs (red) are affected by CHP. Adjusting those IVs would lead to a significant effect estimate of BMI on $\mathrm{TG}, \hat{\beta}_{1(\mathrm{BMI} \rightarrow \mathrm{TG})}=0.262$, and an insignificant reverse causal effect estimate from TG to BMI, $\hat{\beta}_{1(\mathrm{TG} \rightarrow \mathrm{BMI})}=0.008$. In this example, CHP would induce genetic confounding and introduce a significant and negative bias. Using estimated IVs with CHP, one may obtain significant causal and reverse causal effect estimates, $\hat{\beta}_{2(\mathrm{BMI} \rightarrow \mathrm{TG})}=-0.655$, and $\hat{\beta}_{2(\mathrm{BMI} \rightarrow \mathrm{TG})}=-0.443$. (d) An illustration of the MR-CUE model. MR-CUE decomposes IVs into two sets, those not affected by CHP (left, $\eta_{k}=0$ ) and those affected by CHP (right, $\eta_{k}=1$ ). MR-CUE allows all IVs to have potential non-zero UHP effect, $\theta_{k}$. In (d) right panel, we assume that the IV affects the exposure and confounder proportionally, with a sum of IV-to-exposure effect of $\gamma_{k}$. We rescale the IV-to-confounder effect to be 1 and the effect of confounders on exposure is then $\gamma_{k}$ (yellow line). The line representing the direct effect from IV to exposure is omitted since it is assumed to change proportionally with IV-to-confounder effect. The red line represents the decomposed and projected confounder-to-outcome effect and is also proportional to IV-strength, $\gamma_{k}$. The IV-specific perturbation of confounders may induce an IV-specific bias, $\widetilde{\alpha}_{k}$, which has a mean of zero. 


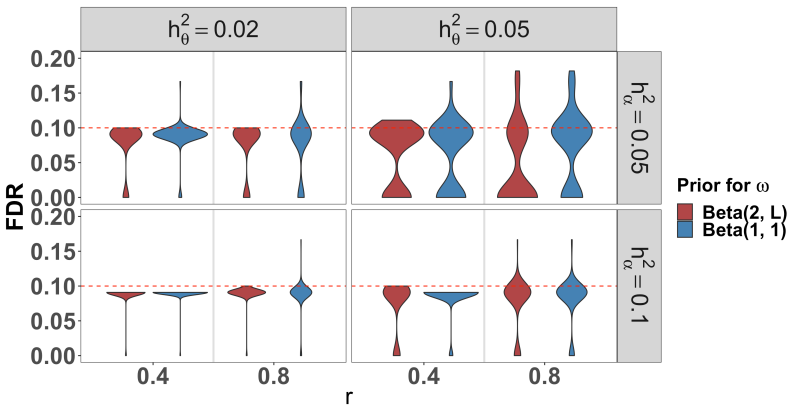

(a) FDR

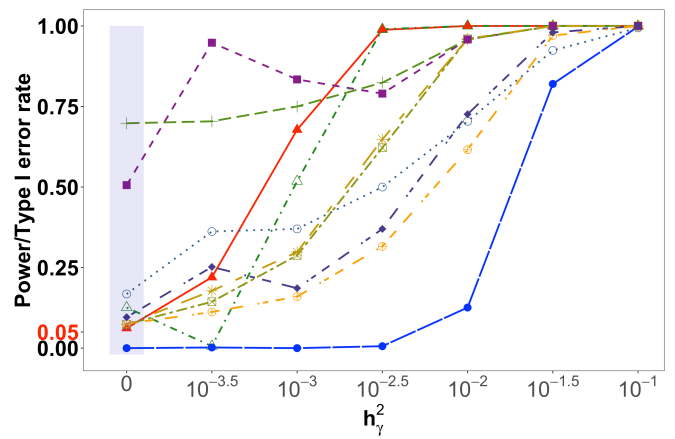

(c) Power/Type I error rate

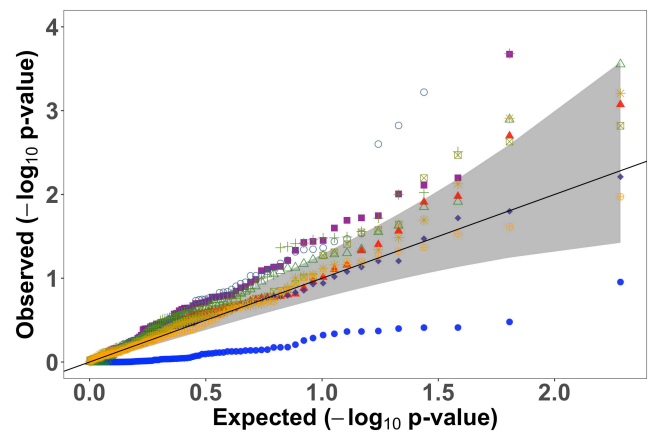

(e) Negative Control

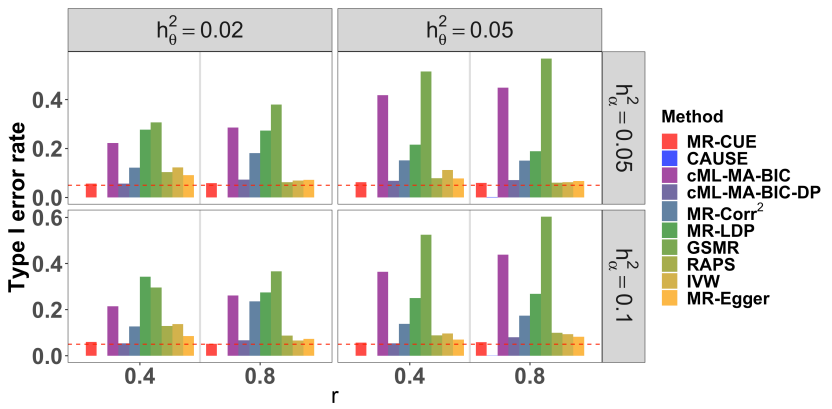

(b) Type I error rate
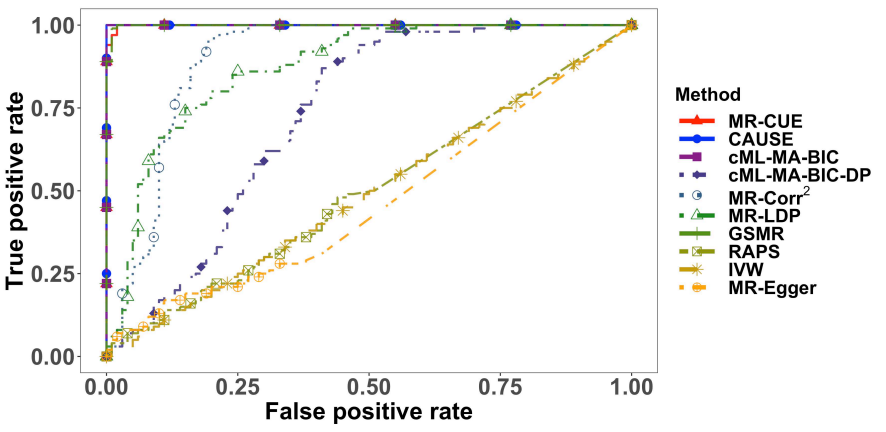

(d) Reverse causation

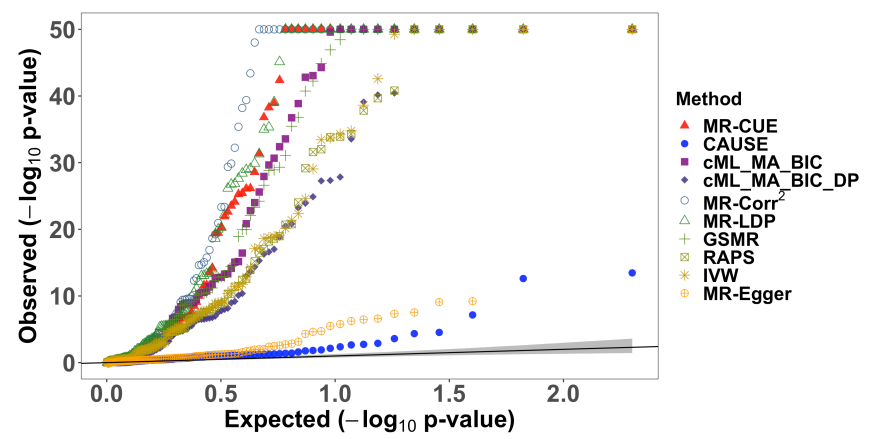

(f) Positive Control

Figure 2: (a) False discovery rates for IVs identified with CHP under combinatorial settings for $h_{\theta}^{2}$ and $h_{\alpha}^{2}$ with $\rho_{\alpha \gamma}=0.2$ and $p=1,000$. (b) Type I error rates for MR-CUE and other methods under combinatorial settings for $h_{\theta}^{2}$ and $h_{\alpha}^{2}$ with $\rho_{\alpha \gamma}=0.2$ and $p=1,000$. (c) Powers for MR-CUE and other methods under the setting: $h_{\theta}^{2}=0.1, h_{\alpha}^{2}=0.05, p=1,000, r=0.4$ and $\rho_{\alpha \gamma}=0.2$. (d) ROC curves for evaluation of causation and reverse causality among all methods. (e) QQ plots of $-\log _{10}$ ( $p$-values) for all methods under the null from analyses of negative controls. (f) QQ plots of $-\log _{10}(p$-values) for all methods from analyses of positive controls. 

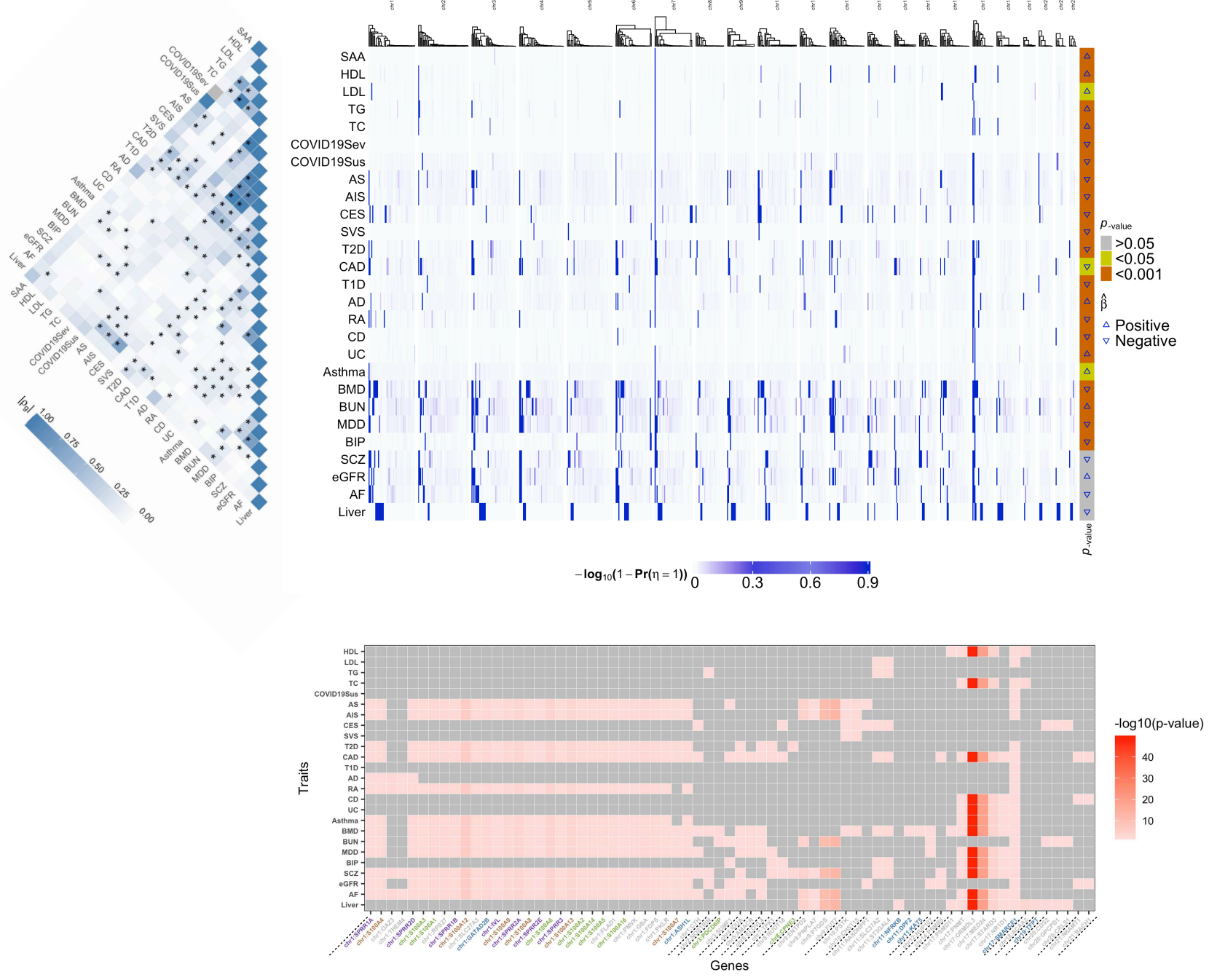

Figure 3: (a) The heatmap of the estimated genetic correlations among the 27 examined outcomes (left panel) with IL-6 as exposure; the heatmap of the estimated strengths of CHP, $-\log _{10}\left(1-\operatorname{Pr}\left(\eta_{l}=1\right)\right)$, for selected IVs across all chromosomes for the 27 outcomes (right panel). (b) The heatmap of a partial list of cis-genes that were significantly associated with at least one IV affected by CHP across multiple outcomes, with color indicating the strength of the most significant association for each gene. Cis-associations were assessed using blood tissue samples from the Genotype-Tissue Expression (GTEx) project for IVs with estimated CHP effect. 


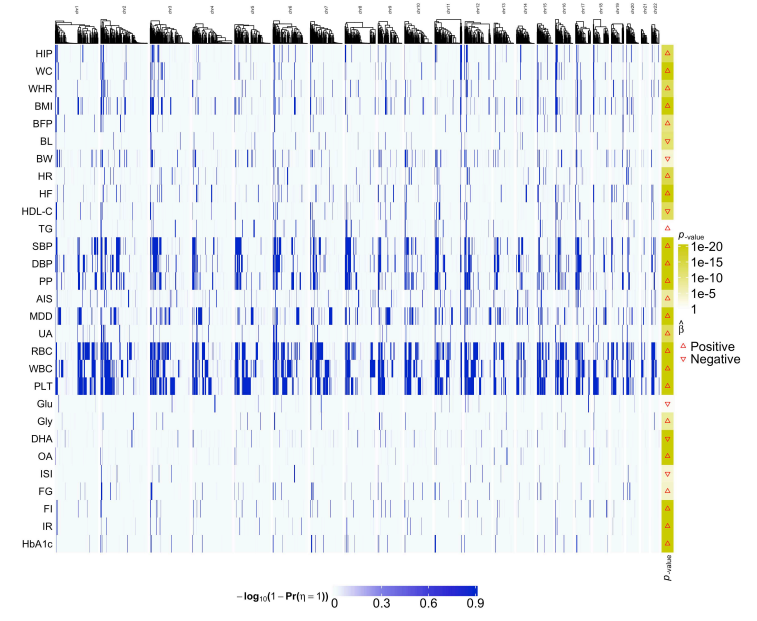

(a) Heatmap for European population

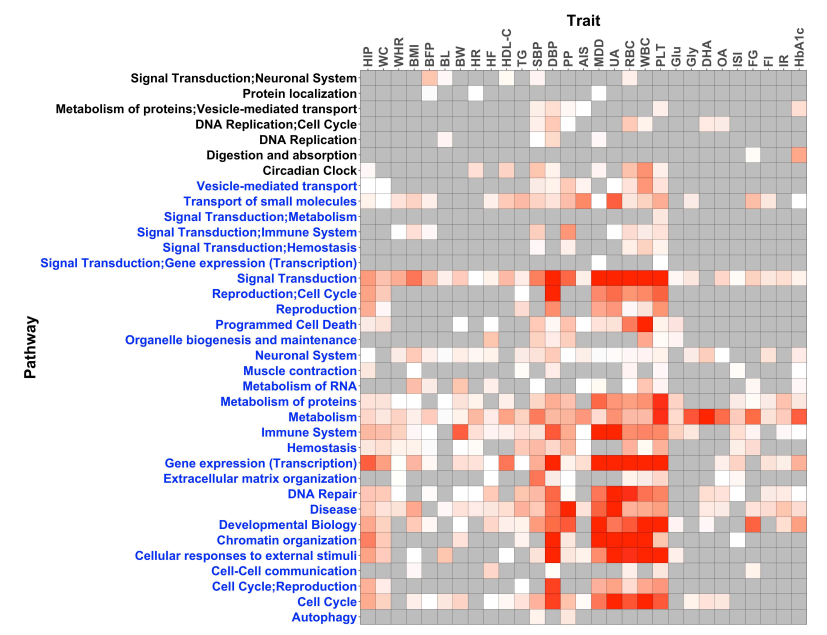

(c) Pathway for European population

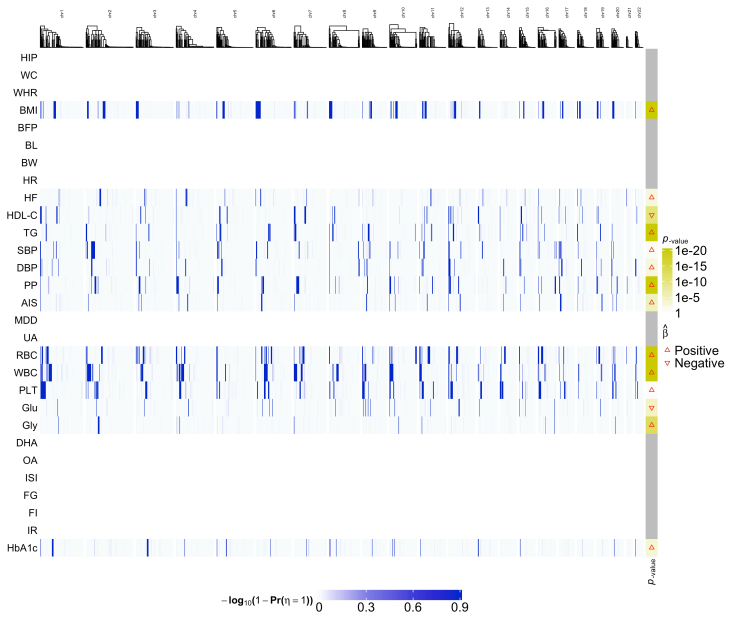

(b) Heatmap for Asian population

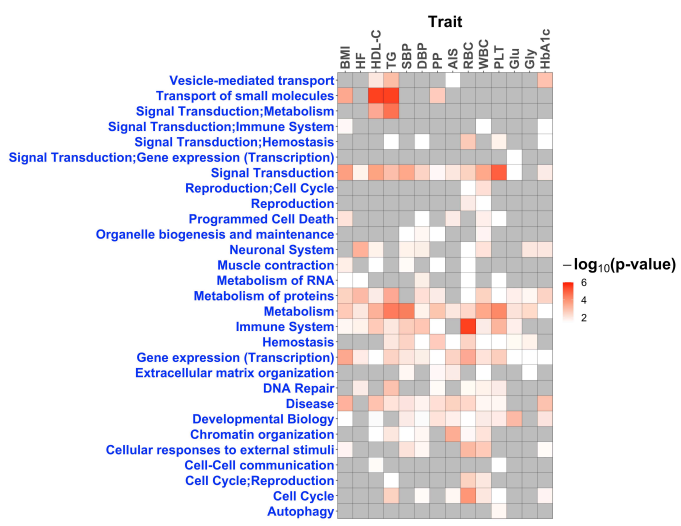

(d) Pathway for Asian population

Figure 4: (a) \& (b) The heatmaps of the estimated strengths of CHP, $-\log _{10}\left(1-\operatorname{Pr}\left(\eta_{l}=1\right)\right)$, for selected IVs across all chromosomes for 29 and 14 exposures for T2D in the European and East Asian populations, respectively. (c) \& (d) The heatmaps of enriched pathways for identified IVs with CHP by exposure in the European and East Asian populations, respectively. 


\section{Supplementary Files}

This is a list of supplementary files associated with this preprint. Click to download.

- MRCuesupp.pdf

- rs.pdf

- SupplementaryData1.xlsx

- SupplementaryData2.xlsx 